\title{
Desempenho de três linhagens de tilápia submetidas ao tratamento com 17- $\alpha$-metiltestosterona em condições ambientais não controladas ${ }^{1}$
}

\author{
Erika Neumann², Teresa Cristina Ribeiro Dias Koberstein², Francisco Manoel de Souza Braga ${ }^{3}$ \\ ${ }^{1}$ Apoio: Coordenação de Aperfeiçoamento de Pessoal de Nível Superior-CAPES. \\ 2 Centro de Aquicultura, Universidade Estadual Paulista - CAUNESP, Jaboticabal, São Paulo, Brasil. \\ ${ }^{3}$ Departamento de Zoologia do Instituto de Biociências, Universidade Estadual Paulista - UNESP, Rio Claro, São Paulo, Brasil.
}

RESUMO - Este estudo foi realizado para descrever o efeito da reversão sexual de linhagens de tilápia sobre as taxas de sobrevivência, o crescimento e o percentual de machos fenotípicos em condições ambientais variáveis. Foram utilizadas duas linhagens de Oreochromis niloticus (tilápia-do-nilo comum e tailandesa) e uma híbrida Oreochromis sp (vermelha), que receberam o hormônio masculinizante 17 - $\alpha$-metiltestosterona incorporado à ração $(60 \mathrm{mg} / \mathrm{kg})$ durante os primeiros 30 dias de vida. Aos 90 dias de idade, a taxa de sobrevivência da tilápia-do-nilo comum superou a da tilápia tailandesa em 39,00\% e da tilápia vermelha em $22,70 \%$, enquanto a taxa instantânea de crescimento foi similar nas linhagens comum e tailandesa e significativamente inferior na linhagem vermelha. O percentual de machos, de acordo com o exame de gônadas, decresceu na seguinte ordem: tilápia-do-nilo comum, tailandesa e vermelha. Em situações de oscilação de temperatura, a linhagem comum tem maior desempenho produtivo, mas são necessários estudos para comprovação da pureza genética e análise do manejo reprodutivo de linhagens de tilápia.

Palavras-chave: crescimento, fator de condição, reversão sexual, sobrevivência

\section{Performance of three tilapia strains submitted to 17- $\alpha$-methyltestosterone, under uncontrolled environmental conditions}

\begin{abstract}
This study describes the differential effect of sexual reversion in tilapia strains on survival rates, growth and the percentage of phenotypical males under different environmental conditions. Two Orechromis niloticus strains (common Nile tilapia and Thai-chiltralada) and one hybrid Oreochromis sp (red tilapia) were used, which were fed from hatch during the first thirty days of life with $17-\alpha$-methyltestosterone hormone incorporated to the ration $(60 \mathrm{mg} / \mathrm{kg})$. At the $90^{\text {th }}$ days, the survival rate of the common Nile had surpassed Thai-chiltralada in $39.00 \%$ and red tilapia in $22.70 \%$, while the growth rate was similar in common Nile and Thai-chiltralada, and slightly lower in red tilapia. The percentage of males decreased at the following order: common Nile, Thai-chiltralada and red tilapia (98.73, 96.23 and $89.46 \%$ respectively. In situations of temperature oscillation, the common Nile strain was more efficient in productive terms; however, further studies to verify the genetic pureness and reproductive management of different strains should be conducted.
\end{abstract}

Key Words: condition factors, growth, sexual reversion, survival rate

\section{Introdução}

O desenvolvimento e a intensificação da tilapicultura dependem do controle de algumas funções fisiológicas, entre elas, a reprodução. A manipulação do sexo fenotípico pelo tratamento de larvas com hormônios masculinizantes é a técnica mais utilizada em peixes para obtenção de populações monossexuais masculinas (Pinto, 1988).

A maior parte dos trabalhos relacionados à reversão sexual enfoca o sucesso ou fracasso dos métodos utilizados, sem adequada atenção às taxas de sobrevivência e crescimento de peixes submetidos ao tratamento hormonal.
Blázquez et al. (1995) e Pandian \& Sheela (1995) afirmaram que peixes sexualmente revertidos podem apresentar baixa sobrevivência e supressão do crescimento, causadas por altas doses de hormônios.

As taxas de sobrevivência e crescimento e o percentual de machos fenotípicos obtidos após o tratamento hormonal de tilápias podem variar entre linhagens. Segundo Rezk et al. (2002), estudos sobre o desempenho de tilápias são voltados à tolerância fisiológica das espécies às variáveis ambientais, de modo que pouca atenção é direcionada ao desempenho de populações submetidas a estas variáveis em cultivo. De acordo com esses autores, comparações 
entre espécies e linhagens de tilápia permitem visualizar como variações genotípicas implicam diferentes respostas em cultivo.

Considerando as significativas diferenças de desempenho entre linhagens de outras espécies de animais domésticos, como aves, suínos e bovinos, e a importância econômica da tilápia-do-nilo para a aquicultura, são necessários estudos para avaliar o desempenho das linhagens desse peixe (Boscolo et al., 2001).

Pesquisadores avaliaram linhagens de tilápia cultivadas no Brasil e comprovaram desempenho diferenciado entre elas quanto ao fator de condição (Boscolo et al., 2001), ao ganho de peso, ao comprimento total final e à taxa de sobrevivência (Tachibana et al., 2004; Wagner et al., 2004), entre outros parâmetros.

Conduziu-se este trabalho com o objetivo de observar respostas intra-específicas para avaliação da efetividade da reversão sexual sobre as taxas de crescimento, sobrevivência e proporção sexual em tilápias mantidas em condições ambientais não controladas, tratadas com $17 \alpha$-metiltestosterona. Foi avaliado o desempenho de três linhagens, duas de Oreochromis niloticus (tilápiado-nilo comum e tailandesa) e uma híbrida Oreochromis sp (tilápia-vermelha).

\section{Material e Métodos}

As coletas foram realizadas no Laboratório de Tilapicultura do Centro de Aqüicultura da Universidade Estadual Paulista - CAUNESP, Jaboticabal, São Paulo.

Três linhagens de tilápia foram estudadas: duas de Oreochromis niloticus, tilápia-do-nilo comum e tilápiatailandesa, e uma híbrida, Oreochromis sp, tilápia-vermelha. Foram acondicionados um macho para 3 fêmeas $/ \mathrm{m}^{3}$ em aquários de $2 \mathrm{~m}^{3}$, com água recirculada e aeração contínua, utilizando-se três aquários para cada linhagem. Após duas semanas de aclimatação, foram coletados (enquanto incubados na boca das fêmeas) seis lotes de ovos de cada linhagem, que foram individualizados em incubadoras de 2 litros de capacidade e fluxo de água contínuo. Após a eclosão, as larvas passaram para bandejas com água filtrada e recirculada, iniciando-se o arraçoamento.

As larvas foram alimentadas quatro vezes ao dia com ração na granulometria de $0,35 \mathrm{~mm}$, contendo $40 \%$ de proteína brutae $60 \mathrm{mg} / \mathrm{kg}$ do hormônio $17-\alpha$-metiltestosterona. A ração foi fornecida à vontade, espalhada sobre a lâmina d'água.

Após oito dias de eclosão, as larvas foram transferidas para aquários de 120 litros, com fluxo de água contínuo, onde ficaram até 15 dias de idade. Posteriormente, foram acondicionadas em tanques-rede (dimensão 2,0 $\mathrm{m} \times 1,0 \mathrm{~m}$ $\times 0,75 \mathrm{~m}$ e malha de $2,0 \mathrm{~mm}$ ), alojados em viveiros escavados de $120 \mathrm{~m}^{2}$ até completarem 30 dias, quando o fornecimento de ração com hormônio masculinizante foi interrompido.

Após o tratamento hormonal dos peixes, os tanquesrede iniciais foram substituídos por outros de $1 \mathrm{~m}^{3}$ e malha de $4 \mathrm{~mm}$, onde os juvenis ficaram até 90 dias de idade, quando foram anestesiados em gelo e fixados em formol a $4 \%$ por 48 horas. A temperatura da água foi monitorada diariamente (termômetro de bulbo de mercúrio de 0 a $50^{\circ} \mathrm{C}$ ) e semanalmente foram feitas análises de $\mathrm{pH}$, condutividade elétrica $\left(\mu \mathrm{S} . \mathrm{cm}^{3}\right)$ e oxigênio dissolvido (mg/L) com analisadores digitais, calculando-se a alcalinidade e amônia pelo método de Goltermann et al. (1978).

As três linhagens de tilápia foram analisadas em delineamento inteiramente casualizado, considerando três tratamentos e seis repetições. De cada amostra fixada aos 90 dias de idade, foi verificado o comprimento total (em estereomicroscópio com ocular micrométrica), ganho de peso (balança analítica de quatro dígitos) e sexo, determinado por exame das gônadas pelo método do acetatocarmin (Wassermann \& Afonso, 2002), de modo que os valores absolutos de indivíduos de cada sexo nas repetições foram transformados em porcentagem.

Os dados de peso $(\mathrm{P})$ e comprimento total $(\mathrm{CT})$ foram ajustados à equação alométrica $\mathrm{y}=\mathrm{ax}{ }^{\mathrm{b}}, \mathrm{em}$ que $\mathrm{y}=\mathrm{P}, \mathrm{x}=\mathrm{CT}$ e $a$ e $b$ estimados pelo método dos mínimos quadrados após transformação dos dados (Vanzolini, 1993).

A alometria e isometria foram definidas pela análise do coeficiente angular $(b)$ na equação alométrica descrita. Para definir a isometria-alometria, foi usada a expressão $\mathrm{t}_{\mathrm{b}}=|\mathrm{b}-3| / \mathrm{S}_{\mathrm{b}}$, em que $b$ é o valor do coeficiente angular e $\mathrm{S}_{\mathrm{b}}$, o desvio de $b$, de modo que $\mathrm{t}_{\mathrm{b}}$ foi comparado em teste bicaudal com n-2 graus de liberdade (Fowler et al., 1998) a fim de verificar se o coeficiente angular foi estatisticamente diferente ou igual a 3.

$\mathrm{O}$ fator de condição alométrico $(\mathrm{K})$ foi analisado entre as linhagens e correspondeu ao valor de $a$ da equação alométrica já descrita (Braga, 1993; 1997). A taxa instantânea de crescimento $(\mathrm{G})$ foi avaliada utilizando-se a fórmula $\mathrm{G}=\ln \mathrm{W} 2-\ln \mathrm{W} 1 / \mathrm{t} 2-\mathrm{t} 1$, em que $\ln \mathrm{W} 2 \mathrm{e} \ln \mathrm{W} 1$ são, respectivamente, os logaritmos dos pesos médios nos instantes t2 (90 dias) e t1 (um dia) (Ricker, 1979).

A sobrevivência dos peixes nas três linhagens de tilápia foi analisada por meio da expressão $S=\mathrm{N}_{\mathrm{t}} / \mathrm{N}_{0}$, em que $\mathrm{N}_{\mathrm{t}}$ é o número total de peixes no instante $\mathrm{t}$ (90 dias), $\mathrm{N}_{0}$ o número inicial total de larvas (oito dias, contadas ao 
serem retiradas da incubadora) e $\mathrm{S}$ a sobrevivência de larvas aos 90 dias de idade (Ricker, 1975).

$\mathrm{Na}$ análise de dados de peso, comprimento total e proporção de sexos, foi aplicada uma descrição de amostras, estimando-se médias e variâncias dos parâmetros analisados para cada linhagem. Em virtude do número de repetições, aplicou-se às variâncias um teste de homogeneidade de Bartlett (Mendes, 1999), seguido do teste Kruskal-Wallis para médias com variâncias heterogêneas e de one-way ANOVA com posterior teste Tukey (Fowler et al., 1998) para médias com diferenças significativas e variâncias homogêneas, adotando-se como significância $\mathrm{P}=0,05$.

\section{Resultados e Discussão}

Valores do coeficiente alométrico (Tabela 1) indicam, em geral, que em juvenis das três linhagens de tilápia a relação entre peso e comprimento total foi alométrica positiva ( $b>3 ; \mathrm{P}<0,05)$, com maior aumento em peso que em comprimento total nesta fase. A alometria negativa não foi observada em nenhuma repetição das linhagens, porém as repetições 4 de tilápia-do-nilo comum, 3 de tailandesa, 5 e 6 de tilápia-vermelha apresentaram isometria, com peso e comprimento total crescendo proporcionalmente. $\mathrm{O}$ grau de correlação entre as variáveis analisadas foi alto e significativo em todos os casos $(r \geq 0,96 ; \mathrm{P}<0,05)$.

$\mathrm{O}$ fator de condição alométrico $(\mathrm{K})$ pelo intercepto (a) diferiu entre repetições de mesma linhagem (Tabela 1) e foi maior nas repetições 4 de tilápia-do-nilo comum, 3 de tilápia-tailandesa, 5 e 6 de tilápia-vermelha, as mesmas com crescimento isométrico na relação entre peso e comprimento total. Nas demais réplicas, esta relação foi alométrica positiva, de modo que o fator de condição diminuiu à medida que os valores numéricos dos coeficientes angulares se distanciaram positivamente de 3 . Segundo Braga (1997), existe relação inversa entre os valores de a (fator de condição) e b da equação alométrica $y=a x^{b}$, ou seja, quanto maior o valor de a, menor o valor de b.

A linhagem vermelha apresentou o maior valor do fator de condição alométrico aos 90 dias, com média de $1,26 \times 10^{-5}$ (Figura 1A). Nas linhagens de tilápia-do-nilo comum e tailandesa, os valores foram similares, com médias de $0,75 \times 10^{-5}$ e de $0,77 \times 10^{-5}$, respectivamente.

As linhagens que tiveram menor valor de fator de condição (Figura 1A) apresentaram maiores taxas de crescimento em peso (Figura 1B), o que corrobora a relação inversa entre fator de condição e coeficiente angular (Braga, 1997). Os valores médios da taxa instantânea de crescimento em tilápia-do-nilo comum, tailandesa e vermelha (Figura 1B) foram de 0,0800; 0,0853 e 0,0726 g/dia, respectivamente.

Os resultados de ganho de peso obtidos neste estudo confirmam os encontrados em outros trabalhos sobre desempenho de tilápia, comprovando que a tailandesa se sobressai à linhagem comum (Boscolo et al., 2001) e à linhagem híbrida (Zanoni et al., 2000; Wagner et al., 2004).

A média de comprimento total da tilápia-tailandesa foi igual às demais, porém as médias de tilápia-do-nilo

Tabela 1 - Dados da relação peso/comprimento observados em alevinos de tilápia aos 90 dias de idade

\begin{tabular}{|c|c|c|c|c|c|}
\hline Linhagem & Repetição & $\mathrm{a}$ & $\mathrm{b}$ & $\mathrm{r}$ & $\mathrm{n}$ \\
\hline \multirow[t]{6}{*}{ Tilápia-do-nilo comum } & 1 & 0,0000044 & $3,3255(\neq 3)$ & $0,97 *$ & 389 \\
\hline & 2 & 0,0000043 & $3,3153(\neq 3)$ & $0,98 *$ & 368 \\
\hline & 3 & 0,0000048 & $3,3032(\neq 3)$ & $0,98 *$ & 330 \\
\hline & 4 & 0,0000210 & $2,9656(=3)$ & $0,98 *$ & 286 \\
\hline & 5 & 0,0000081 & $3,1925(\neq 3)$ & $0,98^{*}$ & 293 \\
\hline & 6 & 0,0000029 & $3,4094(\neq 3)$ & $0,99 *$ & 57 \\
\hline \multirow[t]{5}{*}{ Tilápia-tailandesa ${ }^{1}$} & 1 & 0,0000085 & $3,1707(\neq 3)$ & $0,99 *$ & 45 \\
\hline & 3 & 0,0000140 & $3,0510(=3)$ & $0,98 *$ & 223 \\
\hline & 4 & 0,0000100 & $3,1146(\neq 3)$ & $0,99 *$ & 211 \\
\hline & 5 & 0,0000019 & $3,5547(\neq 3)$ & $0,96^{*}$ & 28 \\
\hline & 6 & 0,0000042 & $3,3631(\neq 3)$ & $0,99 *$ & 74 \\
\hline \multirow[t]{5}{*}{ Tilápia-vermelha ${ }^{1}$} & $1^{2}$ & 0,0000100 & $3,1497(\neq 3)$ & $0,99 *$ & 181 \\
\hline & 3 & 0,0000110 & $3,1378(\neq 3)$ & $0,99 *$ & 361 \\
\hline & 4 & 0,0000110 & $3,1206(\neq 3)$ & $0,99 *$ & 242 \\
\hline & 5 & 0,0000170 & $3,0066(=3)$ & $0,99 *$ & 132 \\
\hline & 6 & 0,0000140 & $3,0521 \quad(=3)$ & $0,98 *$ & 51 \\
\hline
\end{tabular}

$\mathrm{a}=$ valor numérico do intercepto (= fator de condição).

$\mathrm{b}=$ valor do coeficiente angular (estatisticamente $=\mathrm{ou} \neq \mathrm{de} 3$ ).

$\mathrm{r}=$ valor do coeficiente de correlação $(* \mathrm{P}<0,05)$.

$\mathrm{n}=$ número de dados analisados.

${ }_{1}^{1}$ Mortalidade total na repetição 2 de tilápia-tailandesa.

2 Em decorrência de rompimento da malha, as repetições 1 e 2 de tilápia-vermelha foram analisadas conjuntamente. 


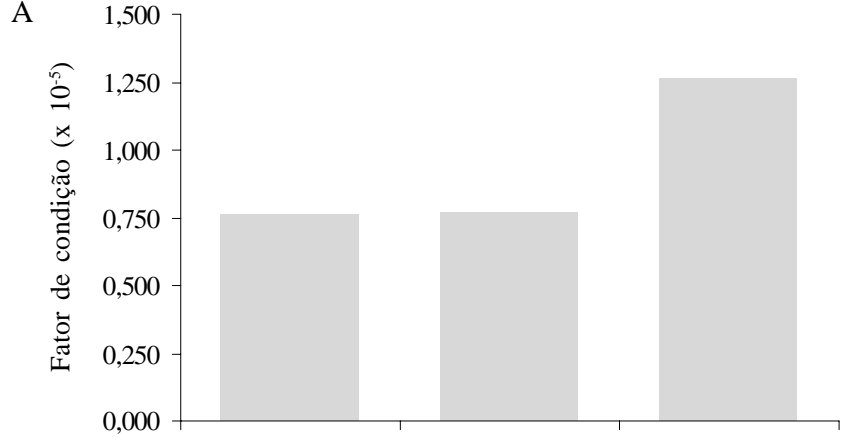

B

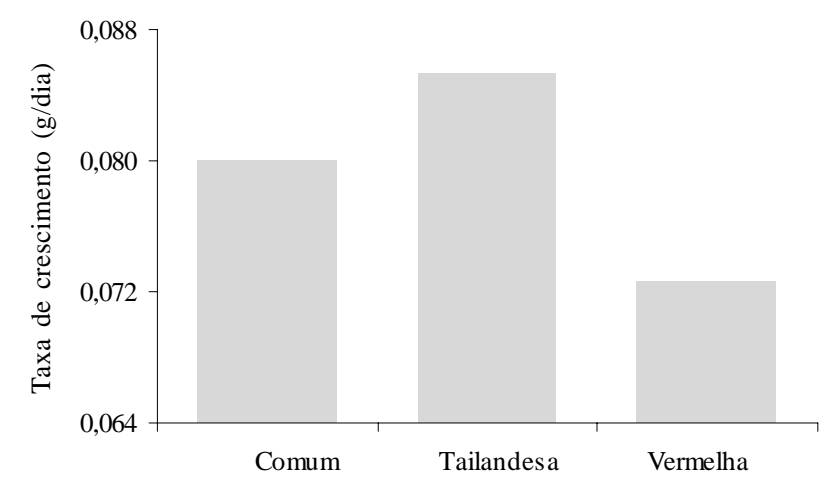

Figura 1 - Fator de condição (A) e taxa instantânea de crescimento (B) médios em juvenis de tilápia-do-nilo comum, tailandesa e vermelha aos 90 dias de idade. comum e vermelha diferiram entre si (Tabela 2). As médias das proporções de machos e de fêmeas diferiram entre as linhagens pelo teste Kruskal-Wallis $(\mathrm{H}=6,45 ; \mathrm{P}<0,05$ para machos e $\mathrm{H}=7,35 ; \mathrm{P}<0,05$ para fêmeas).

Em relação à média do comprimento total, não houve diferença significativa entre as tilápias-do-nilo comum e tailandesa, porém o crescimento da linhagem híbrida foi significativamente menor que o da linhagem comum. Wagner et al. (2004), em análise do desempenho de linhagens de tilápia entre 35 e 87 dias de idade, observaram média de comprimento total significativamente maior na linhagem tailandesa em relação à linhagem de tilápia-do-nilo comum e uma linhagem híbrida. De acordo com esses autores, a semelhança nas médias de comprimento total entre linhagens em algumas fases de desenvolvimento indica que algumas linhagens apresentam crescimento corporal semelhante e diferem exclusivamente pelo peso.

Apesar das boas respostas ao tratamento hormonal pelas linhagens de tilápia-do-nilo comum e tailandesa, a proporção média de machos na linhagem comum foi numericamente superior à da linhagem tailandesa (Tabela 2). Apenas a média de machos de tilápia-vermelha foi relativamente baixa $(89,46 \%)$, embora $90 \%$ de machos ao final da reversão sexual seja um resultado aceitável.

A proporção de intersexos obtida em tilápia-do-nilo comum e tailandesa $(1,16 \mathrm{e} 2,57 \%$, respectivamente) foi mais alta que a de fêmeas (Tabela 2) e só na linhagem vermelha

Tabela 2 - Dados de comprimento total, peso e sexo de alevinos de tilápia aos 90 dias de idade tratados com $17 \alpha$-metiltestosterona

\begin{tabular}{|c|c|c|c|c|c|c|}
\hline Linhagem & Repetição & Comprimento total $(\mathrm{mm})$ & Peso $(\mathrm{g})^{*}$ & Machos (\%) & Fêmeas $(\%)$ & Intersexos* $(\%)$ \\
\hline \multirow[t]{6}{*}{ Tilápia-do-nilo comum } & 1 & 75,74 & 8,05 & 99,49 & 0,00 & 0,51 \\
\hline & 2 & 73,03 & 6,67 & 99,19 & 0,00 & 0,81 \\
\hline & 3 & 80,80 & 9,66 & 98,48 & 0,30 & 1,21 \\
\hline & 4 & 68,44 & 6,16 & 96,85 & 0,00 & 3,15 \\
\hline & 5 & 70,47 & 6,56 & 99,32 & 0,34 & 0,34 \\
\hline & 6 & 86,39 & 11,93 & 98,25 & 0,00 & 1,75 \\
\hline \multirow[t]{5}{*}{ Tilápia-tailandesa } & 1 & 93,95 & 15,99 & 95,56 & 0,00 & 4,44 \\
\hline & 3 & 57,49 & 3,39 & 96,88 & 0,89 & 2,23 \\
\hline & 4 & 71,44 & 6,50 & 97,63 & 0,95 & 1,42 \\
\hline & 5 & 76,65 & 10,38 & 96,55 & 0,00 & 3,45 \\
\hline & 6 & 64,63 & 5,98 & 90,54 & 4,05 & 5,41 \\
\hline \multirow[t]{5}{*}{ Tilápia-vermelha } & 1 & 78,89 & 10,42 & 97,79 & 0,55 & 1,66 \\
\hline & 3 & 50,17 & 2,46 & 75,69 & 17,96 & 6,35 \\
\hline & 4 & 49,12 & 2,31 & 98,35 & 1,24 & 0,41 \\
\hline & 5 & 45,89 & 1,81 & 98,48 & 0,76 & 0,76 \\
\hline & 6 & 57,56 & 3,38 & 92,16 & 1,96 & 5,88 \\
\hline \multirow[t]{3}{*}{ Médias } & Tilápia-do-nilo comum & $75,81 \mathrm{a}$ & 8,17 & $98,73 \mathrm{a}$ & $0,12 \mathrm{c}$ & 1,16 \\
\hline & Tilápia-tailandesa & $72,83 a b$ & 8,45 & $96,23 \mathrm{~b}$ & $1,20 \mathrm{~b}$ & 2,57 \\
\hline & Tilápia-vermelha & $56,33 \mathrm{~b}$ & 4,08 & $89,46 c$ & $7,33 \mathrm{a}$ & 3,20 \\
\hline$\overline{\chi^{2}}$ & & $-13,633$ & $-4,44 * *$ & $8,13 * *$ & 26,76 & 2,57 \\
\hline
\end{tabular}

Letras diferentes em mesma coluna indicam diferença significativa entre médias $(\mathrm{P}<0,05)$ pelos testes Tukey $(\mathrm{CT})$ e Kruskal-Wallis $(\%$ de machos e fêmeas).

* Diferenças não-significativas nas médias entre linhagens $(\mathrm{F}=2,31$ e 1,86 para peso e proporção de intersexo, respectivamente, $\mathrm{P}>0,5)$.

** Variâncias heterogêneas no teste $\mathrm{c}^{2}$ 
foram obtidas mais fêmeas $(7,33 \%)$ que indivíduos intersexuais. Carvalho \& Foresti (1996) observaram que a ocorrência de animais intersexos é maior quando os indivíduos sofreram processo incompleto de reversão sexual, que pode ser causado pela ingestão de quantidade insuficiente de hormônio.

Nas linhagens de tilápia deste estudo, a alta taxa de reversão sexual na maioria das repetições indica que a quantidade de hormônio incorporada à ração foi suficiente para obtenção de taxas de masculinização satisfatórias. $\mathrm{O}$ aparecimento de indivíduos intersexos pode ter sido influenciado pelo fornecimento precoce de hormônio aos peixes, que iniciaram ingestão de ração com andrógeno desde a abertura da boca ( $3^{\circ}$ dia de vida). Bombardelli \& Hayashi (2005) verificaram redução linear no percentual de intersexuais com o aumento da idade inicial ao aplicarem tratamento hormonal por imersão em tilápias e sugeriram que, quanto mais curto e mais próximo do momento de diferenciação sexual for o tratamento, menores as conseqüências metabólicas e a ocorrência de intersexo.

As médias de sobrevivência das três linhagens de tilápia aos 90 dias de idade foram de 58,50; 19,50 e 35,80\%, em tilápias-do-nilo comum, tailandesa e vermelha, respectivamente (Tabela 3). A linhagem de tilápia comum apresentou a maior taxa de sobrevivência e superou em 39,00\% a linhagem tailandesa e em $22,70 \%$ a linhagem vermelha.
As médias da taxa de sobrevivência foram baixas nas três linhagens e podem estar relacionadas ao canibalismo, decorrente da grande heterogeneidade no tamanho dos peixes observada nas réplicas das três linhagens e que resulta no estabelecimento de hierarquias comuns em tilápias (Volpato et al., 1989). Borges et al. (2005) obtiveram taxas de canibalismo de 2,5 a $27,8 \%$ durante a reversão sexual de tilápia, com maiores valores em lotes com maior variação no peso corporal. A queda na temperatura em determinados momentos do desenvolvimento (temperatura mínima abaixo de $20^{\circ} \mathrm{C}$ ) e manejo com trocas freqüentes de ambientes (incubadora $\rightarrow$ aquários $\rightarrow$ tanques-rede de malha pequena $\rightarrow$ tanques-rede de malha grande) ao longo dos 90 dias de cultivo, também podem ter provocado estresse excessivo e contribuído para as baixas taxas de sobrevivência.

Apesar do número variável de indivíduos estocados em cada repetição das linhagens (Tabela 3 ) e da heterogeneidade no crescimento dos peixes em peso e comprimento total (Tabela 2), a densidade de estocagem média das linhagens não correspondeu à taxa de crescimento (Figura 1B), que foi menor na linhagem vermelha, mesmo com menor densidade em comparação à tilápia-do-nilo comum.

Wagner et al. (2004), avaliaram o desempenho produtivo de quatro linhagens de tilápia em condições de cultivo intensivo e observaram diferentes respostas durante o

Tabela 3 - Sobrevivência em linhagens de tilápia-do-nilo comum, tailandesa e vermelha

\begin{tabular}{|c|c|c|c|}
\hline \multirow[t]{2}{*}{ Repetição } & \multicolumn{2}{|c|}{ Número de larvas } & \multirow[t]{2}{*}{ Sobrevivência } \\
\hline & $\begin{array}{l}\text { Início do experimento } \\
\text { (8 dias de vida) }\end{array}$ & $\begin{array}{l}\text { Final do experimento } \\
\text { (90 dias de vida) }\end{array}$ & \\
\hline \multicolumn{4}{|c|}{ Tilápia-do-nilo comum } \\
\hline 1 & 549 & 390 & 0,71 \\
\hline 2 & 462 & 370 & 0,80 \\
\hline 3 & 429 & 330 & 0,77 \\
\hline 4 & 435 & 286 & 0,66 \\
\hline 5 & 625 & 293 & 0,47 \\
\hline 6 & 555 & 57 & 0,10 \\
\hline \multicolumn{4}{|c|}{ Tilápia-tailandesa } \\
\hline 1 & 129 & 45 & 0,35 \\
\hline 2 & 194 & 0 & 0,00 \\
\hline 3 & 1.118 & 224 & 0,20 \\
\hline 4 & 587 & 211 & 0,36 \\
\hline 5 & 254 & 29 & 0,11 \\
\hline 6 & 488 & 73 & 0,15 \\
\hline \multicolumn{4}{|c|}{ Tilápia-vermelha } \\
\hline $1 *$ & 661 & 184 & 0,28 \\
\hline 3 & 561 & 361 & 0,64 \\
\hline 4 & 577 & 241 & 0,42 \\
\hline 5 & 823 & 131 & 0,16 \\
\hline 6 & 178 & 51 & 0,29 \\
\hline
\end{tabular}

* Em decorrência de rompimento da malha, amostras das repetições 1 e 2 de tilápia-vermelha, de mesma idade, estão juntas. 
desenvolvimento, fato que atribuíram ao desempenho não satisfatório da linhagem híbrida, relacionado a sua maior sensibilidade ao sistema de cultivo em comparação às linhagens puras. Mesmo que a menor taxa de crescimento da linhagem vermelha deste estudo tenha sido uma resposta ao sistema de cultivo, possivelmente o crescimento desta linhagem foi menos influenciado pela densidade que pela variação na temperatura da água.

Os valores médios de temperatura $\left(26,09 \pm 2,26^{\circ} \mathrm{C}\right)$, oxigênio dissolvido $(5,69 \pm 1,07 \mathrm{mg} / \mathrm{L}), \mathrm{pH}(6,57 \pm 0,72)$, alcalinidade $(80,14 \pm 41,91 \mathrm{mg} / \mathrm{L})$, amônia $(0,77 \pm 0,52 \mathrm{mg} / \mathrm{L})$ e condutividade elétrica $(97,96 \pm 62,53 \mu \mathrm{S} / \mathrm{cm})$ após retirada das larvas das incubadoras (onde os parâmetros eram constantes) são considerados aceitáveis para o bom desenvolvimento de peixes (Sipaúba-Tavares, 1994).

Apesar das boas médias descritas acima, houve momentos críticos em que a temperatura da água dos viveiros foi inferior a $20^{\circ} \mathrm{C}$, o que inibiu o apetite de larvas que passavam por tratamento hormonal, fato verificado principalmente nos viveiros com larvas da linhagem de tilápiavermelha (observação pessoal). Essa observação pode indicar que esta linhagem é menos tolerante às variações na temperatura da água e que o menor consumo de ração neste momento pode ter induzido a menor taxa de crescimento desta linhagem. A ingestão de menor quantidade de ração pelas larvas da linhagem vermelha em momentos de variação brusca de temperatura implicou menor quantidade de hormônio masculinizante ingerido (uma vez que este hormônio é adicionado à ração), o que pode ter influenciado também a taxa de reversão sexual nesta linhagem, que foi mais baixa.

Variações no desempenho de tilápias, ocasionadas por oscilações na temperatura da água, também foram verificadas por Rezk et al. (2002). Comparando a resposta de espécies e linhagens de tilápias ao declínio na temperatura, esses autores verificaram ganho de peso diferenciado entre os genótipos. Segundo Brett (1979), a taxa de crescimento de uma espécie de peixe em situação de temperatura ótima constante e em outra situação com flutuações de temperatura, ao final de determinado período, pode apresentar médias equivalentes nos dois casos, porém a curva de crescimento, no segundo caso, pode oscilar entre valores extremos, além do limite de tolerância, trazendo consequiências ao crescimento.

\section{Conclusões}

Em condições de cultivo com variação de temperatura não controlada, a linhagem de tilápia-do-nilo comum é a que apresenta melhor desempenho produtivo. A linhagem de tilápia híbrida (vermelha) tem desempenho produtivo inferior e a linhagem tailandesa, desempenho intermediário. São necessários mais estudos para avaliação do desempenho de linhagens de tilápia em diversas condições de manejo e cultivo.

\section{Agradecimentos}

À Coordenação de Aperfeiçoamento de Pessoal de Nível Superior - CAPES, pelo auxílio financeiro concedido na forma de bolsa, e ao Centro de Aquicultura da Universidade Estadual Paulista - CAUNESP, Jaboticabal, SP, por disponibilizar auxílio técnico, local e material biológico utilizados neste estudo.

\section{Literatura Citada}

BRAGA, F.M.S. Análise da equação alométrica na relação peso e comprimento e fator de condição em Plagioscion squamosissimus (Teleostei, Sciaenidae). Revista Brasileira de Biologia, v.7, n.3, p.417-425, 1997.

BRAGA, F.M.S. Análise do fator de condição de Paralonchurus brasiliensis (Perciformes, Sciaenidae). Revista Unimar, v.15, n.2, p.99-115, 1993.

BLÁZQUEZ, M.; PIFERRER, F.; ZANUY, S. et al. Development of sex control techniques for European sea bass (Dicentraechus labrax L.) aquaculture: effects of dietary $17 \alpha$-methyltestosterone prior to sex differentiation. Aquaculture, v.135, p.329-342, 1995.

BOMBARDELLI, R.A.; HAYASHI, C. Masculinização de larvas de tilápia do Nilo (Oreochromis niloticus L.) a partir de banhos de imersão com 17- $\alpha$-metiltestosterona. Revista Brasileira de Zootecnia, v.34, n.2, p.365-372, 2005.

BORGES, A.M.; MORETTI, J.O.C.; McMANUS, C. et al. Produção de populações monossexo macho de tilápia-do-nilo de linhagem chiltralada. Pesquisa Agropecuária Brasileira, v.40, n.2, p.153-159, 2005.

BOSCOLO, W.R.; HAYASHI, C.; SOARES, C.M. et al. Desempenho e características de carcaça de machos revertidos de tilápia do Nilo (Oreochromis niloticus), linhagens tailandesa e comum, nas fases inicial e crescimento. Revista Brasileira de Zootecnia, v.30, n.5, p.1391-1396, 2001.

BRETT, J.R.S. Environmental factors and growth. In: HOAR, W.S.; RANDALL, D.J.; BRETT, J.R. (Eds.) Fish physiology. New York: Academic Press, 1979. v.8, p.599-675.

FOWLER, J.; COHEN, L; JARVIS, P. Practical statistics for field biology. Chichester: John Wiley \& Sons, 1998. 259p.

GOLTERMANN, H.L.; CLYMO, R.S.; OHNSTAD, M.A.M. Methods for physical and chemical analysis of freshwaters. London: Blackweel Science Publication, 1978. 214p. (IBP Handbook, 8).

MENDES, P.P. Estatística aplicada à aquicultura. Recife: Bagaço, 1999. 265p.

PANDIAN, T.J.; SHEELA, S.G. Hormonal induction of sex reversal in fish. Aquaculture, v.138, p.1-22, 1995.

PINTO, C.S.R.M. Criação de tilápias. São Paulo: Instituto de Pesca, 1988. 13p. (Boletim Técnico, 10)

REZK, M.A.; KAMEL, E.A.; RAMADAN, A.A. et al. Comparative growth of Egyptian tilapias in response to declining water temperature. Aquaculture, v.207, p.239-247, 2002.

RICKER, W.E. Computation and interpretation of biological statistics of fish populations. Bulletin of Fishery Research Board, n.191, p.1-382, 1975. 
RICKER, W.E. Growth rates and models. In: HOAR, W.S.; RANDALL, D.J.; BRETT, J.R. (Eds.) Fish physiology, bioenergetics and growth. New York: Academic Press, 1979. v.3, 786p

SIPAÚBA-TAVARES, L.H. Limnologia aplicada à aquicultura. Jaboticabal: Funep, 1994. 70p.

TACHIBANA, L.; CASTAGNOLli, N.; PEZZATO, L.E.; et al. Desempenho de diferentes linhagens de tilápia do Nilo (Oreochromis niloticus) na fase de reversão sexual. Acta Scientiarium, v.26, n.3, p.305-311, 2004

VANZOLINI, P.E. Métodos estatísticos elementares em sistemática zoológica. São Paulo: Hucitec, 1993. 130p.

VOLPATO, G.L.; FRIOLI, P.M.A.; CARRIERI, M.P. Heterogeneous growth in fish: some data in the Nile tilapia Oreochromis niloticus and a general view about the casual mechanisms. Boletim de Fisiologia Animal, v.13, p.7-22, 1989.

WAGNER, P.M.; RIBEIRO, R.P.; MOREIRA, H.L.M. et al Avaliação do desempenho produtivo de linhagens de tilápia do Nilo (Oreochromis niloticus) em diferentes fases de criação. Acta Scientiarium, v.26, n.2, p.187-196, 2004.

WASSERMANN, G.J.; AFONSO, L.O.B. Validation of the acetocarmine technique for evaluating phenotypic sex in nile tilapia (Oreochromis niloticus) fry. Ciência Rural, v.32, n.1, p.133-139, 2002.

ZANONI, M.A.; CAETANO FILHO, M.; LEONHARDT, J.H. Performance de crescimento de diferentes linhagens de tilápiado-nilo, Oreochromis niloticus (Linnaeus, 1758), em gaiolas. Acta Scientiarium, v.22, p.683-687, 2000. 